\title{
Getting rid of the cuckoo Cuculus canorus egg: why do hosts delay rejection?
}

\author{
Anton Antonov, Bård G. Stokke, Arne Moksnes, and Eivin Røskaft \\ Department of Biology, Norwegian University of Science and Technology (NTNU), \\ Realfagbygget, N-7491 Trondheim, Norway
}

\begin{abstract}
Egg discrimination is well documented in many hosts of avian brood parasites, but the proximate mechanisms of egg recognition and rejection decisions are poorly understood. Relevant in this respect is the observation that rejectors of parasite eggs often delay their response. This delay has implications for understanding mechanisms important for egg recognition and is the main focus of the present study. We investigated experimentally the relative effects of egg mimicry and eggshell strength of common cuckoo Cuculus canorus eggs on the delay in rejection in marsh warblers Acrocephalus palustris. In addition, by video recording host responses, we elucidate the proximate mechanisms behind the delayed rejections. Host nests were experimentally parasitized with 3 types of real eggs differing in mimicry and/or eggshell strength. Both egg mimicry and eggshell strength significantly affected the time to rejection, but the effect of mimicry was dominant. The delayed rejection of mimetic eggs was explained by the existence of latency to the release of rejection behavior because of recognition problems. Second, when rejection response towards mimetic eggs was initiated, it was less intense compared with hosts experiencing nonmimetic eggs. Our results are consistent with the hypothesis that host motivation when confronted with mimetic eggs needs to increase above a certain threshold before rejection behavior is released, which likely minimizes the risk of recognition errors. An additional component of the delay in rejection as shown by hosts facing nonmimetic eggs was the seemingly inefficient host rejection behavior, probably reflecting lack of previous experience. Key words: brood parasitism, cuckoo, egg recognition, eggshell strength, marsh warbler, mimicry. [Behav Ecol]
\end{abstract}

\begin{abstract}
$\mathrm{A}^{\mathrm{s}}$ vian obligate brood parasites impose considerable fitness costs on host individuals because the latter often experience a complete loss of reproductive output for the season if parasitized (Øien et al. 1998). Consequently, hosts have evolved a number of anti-parasite defences of which the most ubiquitous and efficient is egg discrimination, that is, recognition and rejection of the parasitic egg (Moksnes et al. 1990; Rothstein and Robinson 1998; Davies 2000). The problem of egg discrimination is most relevant in host-brood parasite systems in which the parasite lays mimetic eggs. The wellknown common cuckoo Cuculus canorus (hereafter cuckoo), for instance, regularly parasitizes about 20 host species in Europe, and with some of these, it has developed remarkably good egg mimicry (Baker 1942; Moksnes and Røskaft 1995; Moksnes et al. 1995).

Egg discrimination is viewed as a decision-making process in which the host responds to cognitive stimuli from the clutch and may also modify its response according to the environmental information of the probability of parasitism (Moksnes, Røskaft, Korsnes 1993; Lindholm 2000; Stokke et al. 2005). Experiments have shown that hosts know the appearance of their eggs and reject foreign eggs no matter if the latter form the minority or the majority of the clutch, that is, egg discrimination is based on true recognition (Rothstein 1975; Davies and Brooke 1989a, 1989b; Moksnes 1992; Lyon 2007). A few host species recognize foreign eggs on the basis of size or shape differences (Mason and Rothstein 1986; Moksnes and Røskaft 1992; Marchetti 2000), but the vast majority respond primarily to deviations in egg coloration (e.g., Davies and Brooke 1988; Rothstein and Robinson
\end{abstract}

Address correspondence to A. Antonov. E-mail: anton.antonov@ bio.ntnu.no.

Received 13 February 2007; revised 27 September 2007; accepted 30 September 2007.

(C) The Author 2007. Published by Oxford University Press on behalf of the International Society for Behavioral Ecology. All rights reserved.

For permissions, please e-mail: journals.permissions@oxfordjournals.org
1998; Antonov, Stokke, Moksnes, Røskaft 2006; Underwood and Sealy 2006). Theoretical models and empirical data suggest that there is a threshold in host cognitive abilities which is optimized by selection along the continuum of egg appearance values so as to maximize the probability that a parasitic egg is identified, whereas minimizing the risk that host own eggs are erroneously rejected in the absence of parasitism, that is, recognition errors (Rothstein 1982; Lotem et al. 1995; Davies et al. 1996; McLean and Maloney 1998; RodríguezGironés and Lotem 1999; Stokke et al. 2007).

Despite considerable research interest, the mechanisms of egg recognition and rejection decisions, however, remain poorly understood (Davies et al. 1996; Rodríguez-Gironés and Lotem 1999; Stokke et al. 2005). A few studies have documented that rejecters often delay their response (Alvarez et al. 1976; Davies and Brooke 1988, 1989a; Lotem et al. 1995), but the significance of this puzzling phenomenon has received little attention. The delay in rejection has implications for the proximate mechanisms of egg recognition and may result from perceptual problems in decision making regarding the foreign egg (Rothstein 1982; Rodríguez-Gironés and Lotem 1999). Indeed, several studies found that mimetic foreign eggs take longer to be rejected than nonmimetic ones (Lotem et al. 1995; Honza, Procházka, et al. 2004; but see Procházka and Honza 2004). In host species that evolved advanced egg discrimination abilities, recognizing a nonmimetic egg should be a straightforward and error-proof task. In this context, quick decision making and release of rejection behavior are expected. On the other hand, as the mimicry of the parasitic eggs improves the ability of the host to recognize it should decrease and the risk of recognition errors should increase (Davies et al. 1996; Rodríguez-Gironés and Lotem 1999; Stokke et al. 2005). In such a context, selection should favor a longer decision-making process during which the host motivation may need to increase above a certain threshold before rejection response is triggered to minimize the risk of errors 
(Rodríguez-Gironés and Lotem 1999). This hypothesized behavioral strategy implies the existence of a latency to the initiation of egg rejection, which might explain why it takes hosts longer time to reject mimetic than nonmimetic foreign eggs. To our knowledge, there are no empirical tests of this scenario.

On the other hand, rejection of even nonmimetic eggs is sometimes delayed in host species which otherwise developed discrimination of such eggs (Lotem et al. 1995, Antonov A, Stokke BG, Moksnes A, Røskaft E, unpublished data; Lindholm 2000), implying that there may be some other factors affecting time to rejection besides mimicry. Brood parasites lay eggs of unusual eggshell strength (Picman 1989; Brooker and Brooker 1991; Picman and Pribil 1997), which may potentially impose purely mechanic difficulties to host species that have relatively small bills and are for this reason constrained to employ puncture ejection as a rejection mode (Rohwer and Spaw 1988; Moksnes et al. 1991; Antonov, Stokke, Moksnes, Kleven, et al. 2006).

Finally, experimental eggs added before the host has completed its clutch take significantly longer to be rejected than those added once the clutch is complete, which indicates that hosts may not inspect their clutches closely enough before clutch completion (Gärtner 1982; Davies and Brooke 1989a; Marchetti 2000; Procházka and Honza 2003; but see Amundsen et al. 2002).

In this paper, we investigate the effects of mimicry and eggshell strength on the time to rejection of foreign eggs in a major host of the cuckoo, the marsh warbler Acrocephalus palustris. In addition, we video recorded host rejection behavior in an attempt to reveal the proximate mechanisms responsible for the delay in rejection. Marsh warblers show well-developed egg recognition abilities and cuckoos parasitizing them often lay mimetic eggs (Moksnes and Røskaft 1995; Antonov, Stokke, Moksnes, Røskaft 2006); thus, we can expect a strong effect of mimicry on the time to rejection. Furthermore, marsh warblers mainly reject cuckoo eggs by puncture ejection (Gärtner 1982; Antonov, Stokke, Moksnes, Kleven, et al. 2006), and the strong eggshell of the cuckoo egg may explain part of the variation in time to rejection. To test the relative effects of mimicry and eggshell strength on the time to rejection, we experimentally parasitized marsh warbler nests with 3 types of foreign eggs differing in these 2 factors: 1) great reed warbler Acrocephalus arundinaceus eggs painted nonmimetic, 2) cuckoo eggs painted nonmimetic in the same way, and 3) unmanipulated cuckoo eggs that looked mimetic in relation to host eggs. Cuckoo and great reed warbler eggs are similar in size, but the former are much more resistant to puncture than the latter (Honza et al. 2001) and marsh warblers experience more difficulties in ejecting cuckoo eggs (Antonov, Stokke, Moksnes, Kleven, et al. 2006). We predicted that 1) nonmimetic cuckoo eggs take longer to be ejected than nonmimetic great reed warbler eggs due to the effects of the stronger eggshell alone. Egg mimicry poses recognition difficulties that are expected to delay rejection further. Hence, we predicted that 2) mimetic cuckoo eggs take longer to be rejected than nonmimetic cuckoo eggs due to the effects of mimicry alone. Furthermore, to test the "increasing motivation" hypothesis (Rodríguez-Gironés and Lotem 1999), we quantified host rejection behavior in the 3 treatments based on video recordings. As marsh warblers are puncture ejectors, rejection behavior is manifested as pecking at the foreign egg. We predicted that 3 ) hosts parasitized with mimetic eggs will start pecking later than those parasitized with a nonmimetic egg.

\section{METHODS}

\section{Study area}

The fieldwork was carried out between 15 May and 20 June in 2005 and 2006 in northwestern Bulgaria between the villages of Zlatia $\left(43^{\circ} 46^{`} \mathrm{~N}, 23^{\circ} 30^{\prime} \mathrm{E}\right)$, Ignatovo $\left(43^{\circ} 46^{`} \mathrm{~N}, 23^{\circ} 28^{\prime} \mathrm{E}\right)$, and Dolni Tsibar $\left(43^{\circ} 48^{\circ} \mathrm{N}, 23^{\circ} 31^{\prime} \mathrm{E}\right)$. More detailed information on the study area and the respective host-brood parasite system can be found elsewhere (Antonov, Stokke, Moksnes, Kleven, et al. 2006; Antonov, Stokke, Moksnes, Røskaft 2006).

\section{Experimental procedure}

Only nests found during nest building or before clutch completion were considered for the experiments. To control for the possibility that time to rejection may be influenced by the timing of parasitism (e.g., Davies and Brooke 1989a; Marchetti 2000), the experiments were started at the end of laying, after the fourth or fifth egg was laid. Marsh warbler clutches consist of 4 or 5 eggs (Cramp 1992, Antonov A, Stokke BG, Moksnes A, Røskaft E, unpublished data). One randomly selected host egg was exchanged with one of the following types of real foreign eggs, representing the 3 experimental treatments: (treatment 1) great reed warbler eggs painted nonmimetic, (treatment 2) cuckoo eggs painted nonmimetic in the same way, and (treatment 3) unmanipulated (unpainted) cuckoo eggs that looked mimetic in relation to host eggs (see below). Great reed warbler eggs were used as a control egg type to account for the extent to which the increased eggshell strength of the cuckoo egg may cause a delay in rejection (see Honza et al. 2001; Antonov, Stokke, Moksnes, Kleven, et al. 2006). Only unincubated foreign eggs (estimated by use of floating test, see Hays and LeCroy 1971) were used because eggshell thickness and strength decrease during the course of incubation (Rothstein 1972).

Most experimental foreign eggs were collected from naturally parasitized great reed warbler or marsh warbler nests from the same area, which were either deserted in response to parasitism or multiply parasitized. Cuckoo eggs laid in the nests of marsh warbler and great reed warbler are similar in appearance (Baker 1942; Moksnes and Røskaft 1995, Antonov A, Stokke BG, Moksnes A, Røskaft E, unpublished data). Rejection rate of experimentally introduced real cuckoo eggs in this study (64\%, Antonov A, Stokke BG, Moksnes A, Røskaft E, unpublished data) was not significantly different from the rate at which marsh warblers rejected naturally laid cuckoo eggs in the same area $\left(50 \%, \chi^{2}=0.47\right.$, degree of freedom $[\mathrm{df}]=1$, $P=0.49$; Antonov, Stokke, Moksnes, Røskaft 2006).

Experimental eggs of the 2 painted treatments were uniformly and densely covered with black spots by using indelible ink pen so that little of the background was left visible. After painting, these eggs attained identical appearance, looking very dissimilar to host eggs, and we refer to them as nonmimetic (Figure 1). The mimicry of unpainted cuckoo eggs was assessed visually by 4 test persons on photos of the clutches on a scale from 1 (perfect mimicry) to 5 (no mimicry) (Moksnes, Røskaft, Bičík, et al. 1993). The test persons were consistent in scoring mimicry (repeatability $=0.62, F_{13,42}=7.61, P<0.001$ ), justifying using the mean of the 4 scores. Unpainted cuckoo eggs were on average rather good mimics of host eggs, and we refer to them as mimetic (mimicry $x=2.2 \pm 0.6$, Figure 1 ).

After the start of the experiment, each nest was visited daily until hatching to ascertain if the foreign egg was rejected or accepted. Only responses by rejection (ejection or desertion) are considered here. Rejection mode in all the cases was puncture ejection, and it was manifested by the disappearance of the foreign egg with or without damage of the host eggs (i.e., ejection costs).

Treatments (1) and (2) were applied in both 2005 and 2006, whereas treatment (3) was added in 2006. There were no significant between-year differences in the time to rejection within either painted great reed warbler (Mann-Whitney $U$ test, $U=79.0, P=0.30, n_{1}=11, n_{2}=17$ ) or painted cuckoo 


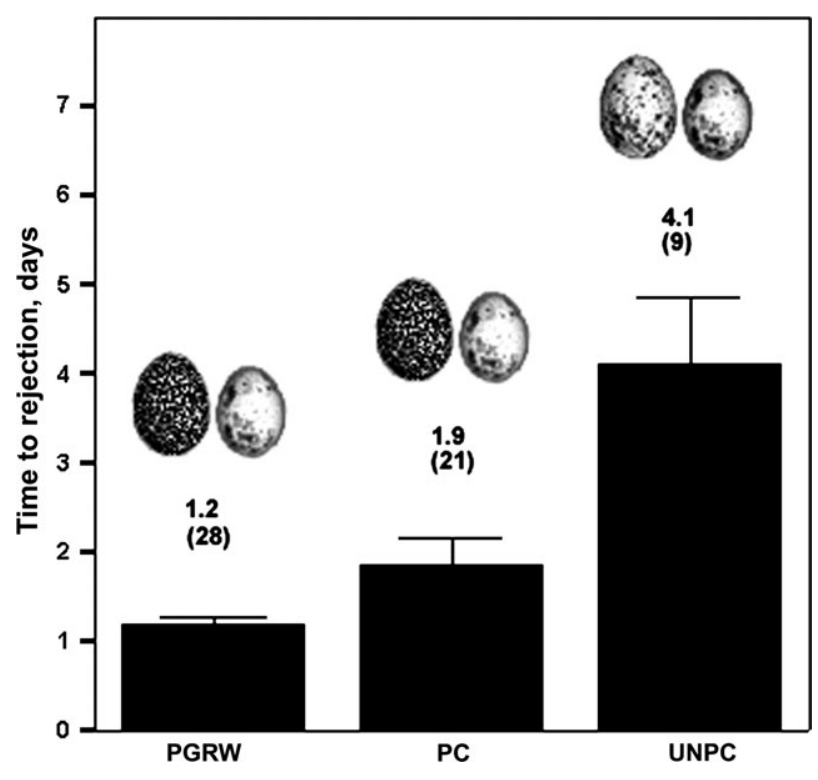

Figure 1

Time to rejection in marsh warblers in relation to 3 types of experimental foreign eggs differing in mimicry and eggshell strength. PGRW, painted great reed warbler egg; PC, painted cuckoo egg; and UNPC, unpainted cuckoo egg. Numbers above the bars are means (sample sizes). Treatment significantly affected rejection time (Kruskal-Wallis ANOVA: $\chi^{2}=24.496, \mathrm{df}=2, P<0.001$ ), and differences were significant between each pair of treatments (Mann-Whitney tests, all $P<0.05$ ).

egg treatment $\left(U=37.0, P=0.16, n_{1}=11, n_{2}=10\right)$. Therefore, we combined the data from the 2 years. In total, we could use the data for 28 rejections of painted great reed warbler eggs, 21 for painted cuckoo eggs, and 9 for unpainted cuckoo eggs.

There is indirect evidence that older females in the congeneric great reed and reed warbler Acrocephalus scirpaceus start breeding earlier than younger ones and in the reed warbler that older females also lay larger clutches (Lotem et al. 1992, 1995; Øien et al. 1996). In addition, rejection rate of foreign eggs seems positively associated with age/experience in the great reed warbler (Lotem et al. 1992, 1995). Thus, age may potentially be expected to affect the delay to rejection as well. Considering painted great reed warbler and painted cuckoo egg treatments, year had a significant effect on laying dates $\left(F_{1,45}=8.78, P=0.05\right)$, the latter being significantly earlier in 2006 than in 2005 . However, treatment $\left(F_{1,45}=0.16, P=0.69\right)$ and the interaction of treatment and year had no significant effect on laying dates $\left(F_{1,45}=0.02, P=0.88\right)$. Furthermore, clutch size was not affected by year $\left(F_{1,45}=0.03, P=0.86\right)$, treatment $\left(F_{1,45}=0.49, P=0.49\right)$, or their interaction $\left(F_{1,45}=\right.$ $2.51, P=0.12)$. Finally, in 2006 when the unpainted cuckoo egg treatment was applied as well, mean laying date and clutch size did not differ significantly among the 3 treatments (laying date: $F_{2,33}=1.79, P=0.18$; clutch size: $F_{2,33}=1.38, P=$ $0.27)$. If laying date and/or clutch size are correlated with age in the marsh warbler, then we can assume no betweentreatment differences in the proportion of older versus younger breeders.

\section{Video recording procedure and behavioral variables}

Video recording equipment included Sony MV 450i video cameras and DVM80 videocassettes. The cameras were mounted on a tripod placed at about $1.5 \mathrm{~m}$ from the nest and above its level to allow close monitoring of host behavior. Most marsh warbler pairs accepted video recording equip- ment and returned to their nests within 2-20 min after the start of the experiment. These intervals are within the normal range of off-nest bouts during incubation in this species (Cramp 1992).

Each nest was video recorded daily for $2 \mathrm{~h}$ for a period of up to 4 days if the foreign egg was not rejected within this interval. The first video recording session started immediately after the experimental egg was introduced in the nest. Timing of the video recordings was randomized among nests and time of the day. It was shown in the related reed warbler that nest attendance and clutch inspection behavior are not influenced by the time of the day (Moksnes et al. 2000; Honza, Grim, et al. 2004).

Logistic and time constraints prevented us from video recording all experimental nests. In total, we were able to use video recording data for 14 nests experimentally parasitized with painted great reed warbler eggs, 13 with painted cuckoo eggs, and 7 with unpainted cuckoo eggs. For 1 nest in the painted great reed warbler treatment and 2 nests in the painted cuckoo egg treatment, we could not ascertain the final response to the foreign egg because these nests were predated. However, these pairs showed pecking on the first video recording day and were included in the analyses. This is justified because in the nests with known responses to the foreign eggs, the presence of pecking always led to subsequent rejection, whereas its absence always led to acceptance (Antonov A, Stokke BG, Moksnes A, Røskaft E, unpublished data, see also Soler et al. 2002).

On each tape, we quantified the amount of time a host was incubating, inspecting or moving the eggs, pecking at the foreign egg or being away from the nest. Recording of behavioral data started when the host appeared at the nest after the start of the experiment. Marsh warblers reject cuckoo eggs by puncture ejection; thus, we considered pecking as the only unequivocal manifestation of egg discrimination. At least in the study population, egg pecking can be regarded as evidence for recognition and a certain predictor of subsequent rejection (Antonov A, Stokke BG, Moksnes A, Røskaft E, unpublished data, see also Soler et al. 2002). We were only interested in between-treatment differences in egg pecking; thus, we did not use a control treatment of nests receiving no foreign eggs.

We considered the following 3 aspects of pecking behavior:

1. Pecking rate-The total number of pecks divided by the total amount of time there was a host at the nest. The more time a host spends at the nest, the greater the expected number of pecks; hence, pecking rate is a more correct representation than the number of pecks itself. For convenience, pecking rate is expressed as number of pecks $\times$ hour $^{-1}$.

2. Pecking strength-Preliminary inspections of video recordings showed that pecking differed substantially in the effort exerted by the host and that a lot of pecks were too weak to result in puncture. Therefore, we quantified pecking strength by scoring pecks according to the following scale-1, weak: very soft pecking producing no tapping sounds, the amplitude of the bill tip is very close to the egg surface, and involves only head and neck movements; 2, intermediate: appreciably more vigorous pecking of higher amplitude but still no tapping sounds or barely audible; 3 , strong: powerful pecking blows producing clear tapping sounds and involves movements of the whole body to increase the amplitude; and 4, very strong: heaviest pecking involving the host literally thrusting itself against the foreign egg, accompanied with wing flapping, very loud tapping sounds produced, and always performed from the nest rim and/or supporting vegetation, obviously to maximize the amplitude of 
pecking. For clarity and illustrative purposes in some parts of the paper, we categorize pecks as weak (scores 1 and 2) and strong (scores 3 and 4).

3. Pecking time-The proportion of time spent pecking of the total amount of time there was a host at the nest, expressed as a percentage.

Most of the rejection events took place later than $2 \mathrm{~h}$ after the start of the experiment, and many of these did not take place until after $24 \mathrm{~h}$ (see Results). For this reason, with a few exceptions, we were unable to video record host behavior continuously until rejection. We thus resorted to comparisons of host pecking behavior among treatments on the first video recording day and on the day first pecking was observed. For the 2 painted treatments, the day of first pecking was always the first day (see Results).

Activities other than pecking were considered only insofar as they could bias the occurrence of pecking among treatments. The 3 treatments did not differ in the proportion of time there was a host at the nest (first day: Kruskal-Wallis ANOVA, $\chi^{2}=1.44, \mathrm{df}=2, P=0.49$; the day first pecking was observed: $\chi^{2}=1.17$, df $\left.=2, P=0.56\right)$. Because the time spent incubating was strongly correlated with the total time spent at the nest $(r=0.99, P<0.0001, n=34)$, we do not consider the former as long as the latter was accounted for. Furthermore, the proportion of time hosts were looking at the eggs or moving them of the total time a host was at the nest did not differ significantly among treatments (first day: $\chi^{2}=$ $1.22, \mathrm{df}=2, P=0.54$; the day the first pecking was observed: $\left.\chi^{2}=0.81, \mathrm{df}=2, P=0.67\right)$. Therefore, the occurrence and intensity of pecking in the 3 treatments was not biased by differences in the total amount of time hosts spent at the nest or viewing the eggs.

Due to the lack of marked and sexed birds, we could not ascertain the sex responsible for pecking and rejection, but given that both sexes take an equal share in incubation (Cramp 1992), both are expected to participate in pecking (Soler et al. 2002). In the related reed warbler and also in 2 Sylvia warblers in which both sexes incubate, both pair members pecked experimental eggs (Davies and Brooke 1988; Soler et al. 2002). We assume that there are no reasons to expect systematic differences in male/female contribution to pecking in the 3 treatments.

\section{Statistical analyses}

Statistical procedures were performed using SPSS 14.0 (SPSS Inc., 2005). The time to rejection and pecking variables were not normally distributed and could not be transformed to achieve normality. For this reason, we used nonparametric tests such as Kruskal-Wallis ANOVAs and Mann-Whitney tests to investigate differences among the experimental treatments (Zar 1999). Following Nakagawa (2004), we did not apply Bonferroni correction for multiple comparisons when all variables were significant at the 0.05 level. Average values are reported as means \pm standard errors. All tests are 2-tailed unless stated otherwise.

\section{RESULTS}

\section{Time to rejection in relation to treatment}

Experimental treatment significantly affected time to rejection, the latter increasing in the sequence as follows: painted great reed warbler, painted cuckoo, and unpainted cuckoo egg treatment (Figure 1). As much as $30 \%$ (3/9) of unpainted cuckoo eggs were rejected after $6-8$ days (Table 1 ). There was a significant correlation between mimicry of the foreign egg and time to rejection within the unpainted cuckoo egg treatment $\left(r_{\mathrm{s}}=-0.68, P=0.04, n=9\right)$. Even though the 2 non-
Table 1

Distribution of the experimental marsh warbler clutches in relation to the time to rejection (in days)

\begin{tabular}{|c|c|c|c|c|c|c|c|c|c|}
\hline \multirow[b]{2}{*}{ Treatment } & \multicolumn{8}{|c|}{ Days } & \multirow[b]{2}{*}{ Total } \\
\hline & 1 & 2 & 3 & 4 & 5 & 6 & 7 & 8 & \\
\hline PGRW & 23 & 5 & 0 & 0 & 0 & 0 & 0 & 0 & 28 \\
\hline PC & 11 & 6 & 3 & 0 & 0 & 0 & 1 & 0 & 21 \\
\hline UNPC & 0 & 3 & 2 & 0 & 1 & 2 & 0 & 1 & 9 \\
\hline Total & 34 & 14 & 5 & 0 & 1 & 2 & 1 & 1 & 58 \\
\hline
\end{tabular}

PGRW, painted great reed warbler egg; PC, painted cuckoo egg; and UNPC, unpainted cuckoo egg.

mimetic egg types were rejected quicker than the mimetic (unpainted) egg type, $17.9 \%(5 / 28)$ of painted great reed warbler eggs and $47.6 \%(10 / 21)$ of painted cuckoo eggs were rejected after 2 or more days (Table 1 ).

Time to rejection differed significantly in relation to treatment in the subsample of video recorded nests as well (Kruskal-Wallis ANOVA, $\chi^{2}=16.54$, df $=2, P<0.001$ ), and differences were also significant between each 2 treatments (Mann-Whitney tests, all $P<0.05$ ).

\section{Egg pecking behavior}

The latency to first pecking varied among the treatments. All host pairs experimentally parasitized with painted great reed warbler or painted cuckoo eggs $(n=27)$ pecked at the foreign egg during the first $2 \mathrm{~h}$ of the experiment. In as much as $70.3 \%(19 / 27)$ of the cases, first pecking was observed as soon as the birds arrived at the nests. In the unpainted cuckoo egg treatment, however, only 1 out of the $7(14 \%)$ of the filmed host pairs showed pecking within the first video recording session, which is significantly lower than for painted eggs (Fisher's Exact test, $P<0.001$ ).

Furthermore, $11 \%(3 / 27)$ of the pairs presented with painted eggs rejected them within the first $2 \mathrm{~h}$, whereas no unpainted cuckoo eggs were rejected sooner than 2 days after the onset of the experiment. Within the unpainted cuckoo egg treatment, there was a significant positive correlation between the day the first pecking was observed and the day of rejection $\left(r_{\mathrm{s}}=0.81, n=7, P=0.03\right)$, that is, the sooner we observed pecking, the sooner the egg was rejected. However, controlling for the day the first pecking was observed, there were no significant differences in the time to rejection between the unpainted and painted cuckoo egg treatment (Mann-Whitney test, $U=38.0, P=0.96, n_{1}=11, n_{2}=7$ ).

When we compared pecking behavior between the 3 treatments on the day the first pecking was observed, the following pattern was evident. The type of the experimental egg influenced pecking rate (Kruskal-Wallis ANOVA, $\chi^{2}=9.19, \mathrm{df}=2$, $P=0.01)$, pecking strength $\left(\chi^{2}=6.58, \mathrm{df}=2, P=0.04\right)$, and pecking time $\left(\chi^{2}=10.35\right.$, df $\left.=2, P=0.01\right)$. Painted great reed warbler and painted cuckoo egg treatments did not differ in any of the 3 variables. However, pecking rate, pecking strength, and pecking time were significantly lower in the unpainted cuckoo egg treatment than in each of the painted egg treatments (Figure 2).

Nevertheless, even hosts confronted with painted eggs showed a generally low pecking effort. Such hosts pecked the foreign egg on average $276.7 \pm 77.2$ times during the 2-h video recording session, but as much as $89 \%$ of these pecks scored weak. In fact, $70 \%(19 / 27)$ of the pairs showed only weak pecking, and the remaining $30 \%$ (8/27) showed strong pecking as well. The latter were significantly more likely to 

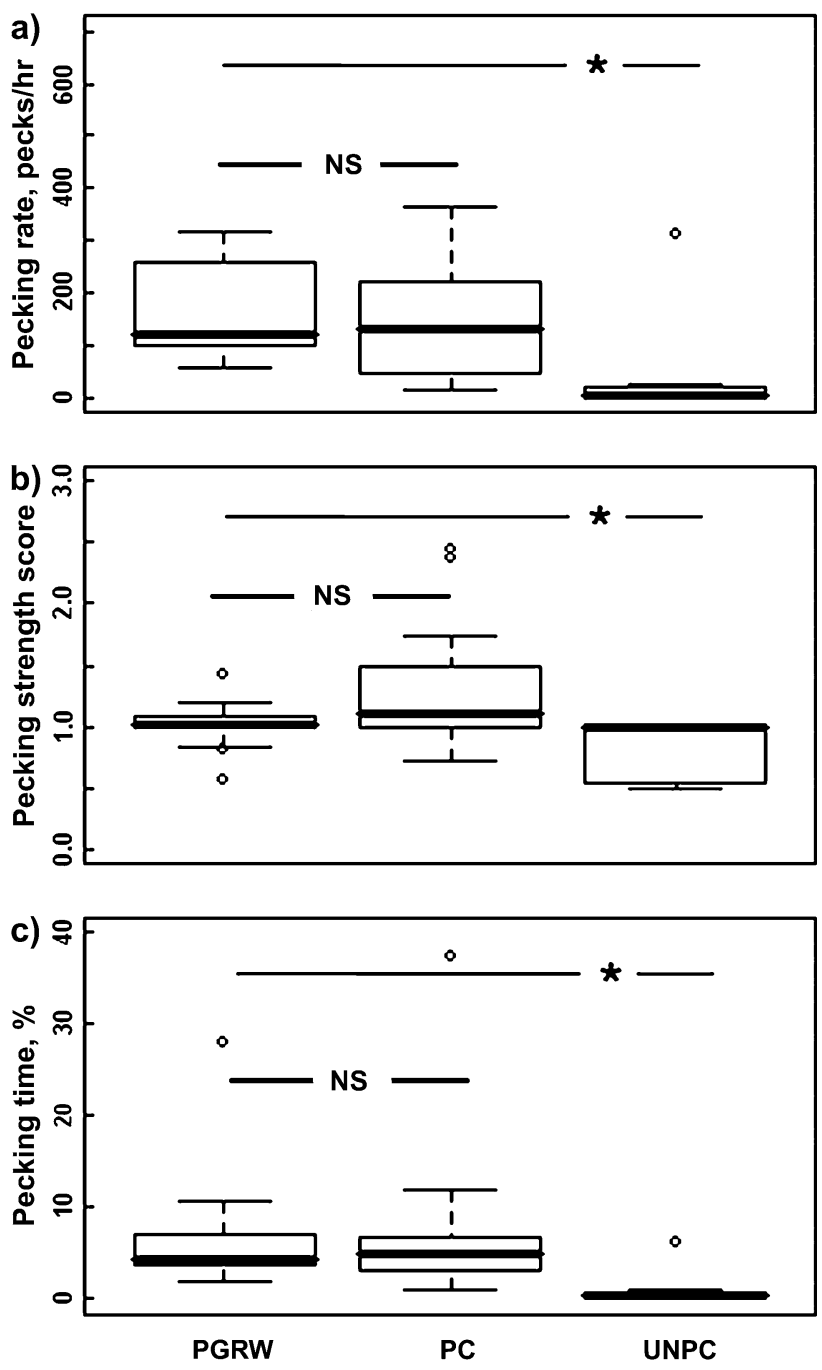

Figure 2

Marsh warbler pecking behavior in relation to 3 types of foreign eggs differing in mimicry and eggshell strength during a 2-h video recording session on the day the first pecking was observed. PGRW, painted great reed warbler egg; PC, painted cuckoo egg; and UNPC, unpainted cuckoo egg. NS means nonsignificant, whereas "*” indicates statistical significance $(P<0.05)$. (a) Pecking rate, $(\mathrm{b})$ pecking strength-assessed on a scale from 1 (weak pecking) to 4 (very strong pecking), (c) pecking time-the proportion of the time spent pecking in relation to the total time a host was at the nest. PGRW and PC did not differ significantly in any of the 3 variables (Mann-Whitney $U$ tests, all $P>0.05$ ), but each of these treatments had significantly higher medians than UNPC $(P<0.05)$.

reject the painted foreign egg during the first $2 \mathrm{~h}$ of the experiment than pairs showing no strong pecking $(3 / 8$ vs. $1 / 19$, Fisher's Exact test, $P=0.02$ ).

Puncture and ejection of painted eggs was documented in 6 cases (3 great reed warbler eggs: on the first, second, and third days, respectively, and 3 cuckoo eggs: 2 on the first day and 1 on the third day). The pecking stints during which the foreign egg was punctured lasted on average $31.7 \pm 7.97 \mathrm{~s}$ and $95.0 \pm$ $33.71 \mathrm{~s}$ for the great reed warbler and cuckoo eggs, respectively (Mann-Whitney test, $W=2, P=0.20, n_{1}=3, n_{2}=3$ ). Regarding these pecking stints, it took the host on average only $20.0 \pm 7.23$ and $54.3 \pm 10.74$ strong pecks to puncture a great reed warbler and a cuckoo egg, respectively ( $W=0$, $P=0.05, n_{1}=3, n_{2}=3$, 1-tailed). The corresponding figures regarding rejections within the first $2 \mathrm{~h}$ only were as follows: for the single great reed warbler egg, $37 \mathrm{~s}$ and 32 pecks, and for the 2 cuckoo eggs, $127.5 \pm 12.7 \mathrm{~s}$ and $55.5 \pm 15.1$ pecks. Nevertheless, rejecting hosts had produced on average $920.7 \pm$ 600.91 and $223.7 \pm 124.34$ weak pecks at the great reed warbler and cuckoo eggs, respectively, during the same 2-h video recording session. The predominance of weak pecks in some of these pairs is depicted in Figure 3.

\section{DISCUSSION}

\section{Time to rejection in relation to treatment}

The time it took the hosts to reject the experimental egg was affected by treatment. In support to prediction (1), painted cuckoo eggs were ejected significantly later than painted great reed warbler eggs. The mimicry was controlled by painting in both treatments, and hosts responded to both types of eggs by pecking more or less immediately; therefore, the delayed rejection of cuckoo eggs must be attributable to the greater difficulties associated with puncturing them. Cuckoo and great reed warbler eggs are similar in size, but the former are much more resistant to puncture than the latter (Honza et al. 2001; Antonov, Stokke, Moksnes, Kleven, et al. 2006) and thus require more effort to be punctured. This is also supported by the fact that marsh warblers rejecting cuckoo eggs are significantly more likely to damage some of their own eggs (ejection costs) than those ejecting great reed warbler eggs (Antonov, Stokke, Moksnes, Kleven, et al. 2006). Moreover, the data from the 6 video recorded rejections provided additional and direct evidence that cuckoo eggs need more pecking effort to be punctured (but see below). The strong eggshell of the cuckoo egg alone, however, caused a relatively short delay ( 0.7 days) in rejection in relation to a painted great reed warbler egg, that is, a control egg of "normal" eggshell strength.

On the other hand, mimicry of the foreign egg had a much stronger effect on the time to rejection because unpainted (mimetic) cuckoo eggs were rejected on average 2.2 days later than painted (nonmimetic) cuckoo eggs, supporting prediction (2). Moreover, the effect of mimicry was strong as was evident by the significant correlation between mimicry and time to rejection within the unpainted cuckoo egg treatment, despite the relatively small sample size. This is to be expected because marsh warblers have well-developed egg recognition abilities, responding to deviations in mimicry and rejecting even some experimentally introduced conspecific eggs (Antonov, Stokke, Moksnes, Røskaft 2006). In conclusion, the joint effects of eggshell strength and mimicry of the cuckoo egg on the delay in rejection was 2.9 days in relation to a thinshelled and nonmimetic control egg. The delay was, however, mainly brought about by mimicry.

The information on the time to rejection brings additional insight into the tolerance to foreign eggs besides rejection rates (Rothstein 1982). The effect of mimicry on the time to rejection documented here is in agreement with the results for other Acrocephalus warblers (e.g., Davies and Brooke 1988; Lotem et al. 1995). However, in 2 other ejector species, whitethroats Sylvia communis and yellowhammers Emberiza citrinella, no significant difference was evident in the time to rejection of nonmimetic and mimetic (conspecific) eggs, both egg types being rejected at a high rate, generally within a day (Procházka and Honza 2003, 2004). There is indirect evidence that cuckoos abandoned these hosts because they have evolved extraordinarily good egg recognition abilities and very high rejection rates of foreign eggs as a consequence (Moksnes and Røskaft 1995; Procházka and Honza 2003, 2004). Warblers of the genus Acrocephalus do not exhibit such advanced anti-parasite defences and are currently among the major cuckoo hosts in Europe. 


\section{Figure 3}

Pecking profiles of marsh warblers at 4 nests that punctured the foreign painted cuckoo or great reed warbler egg during a 2-h video recording session. The origin of the $x$ axis marks the time a host arrived at the nest. Pecking strength was assessed on a scale from 1 (weak pecking) to 4 (very strong pecking). $N$ is the total number of pecks during the video recording session. Bubble size and associated numbers represent the number of pecks during the respective pecking stint. Last bubble marks the time when the egg was punctured. Maximum pecking strength during the last pecking stint preceding puncture may not be evident because a pecking stint may include both weak and strong pecks and mean values are shown

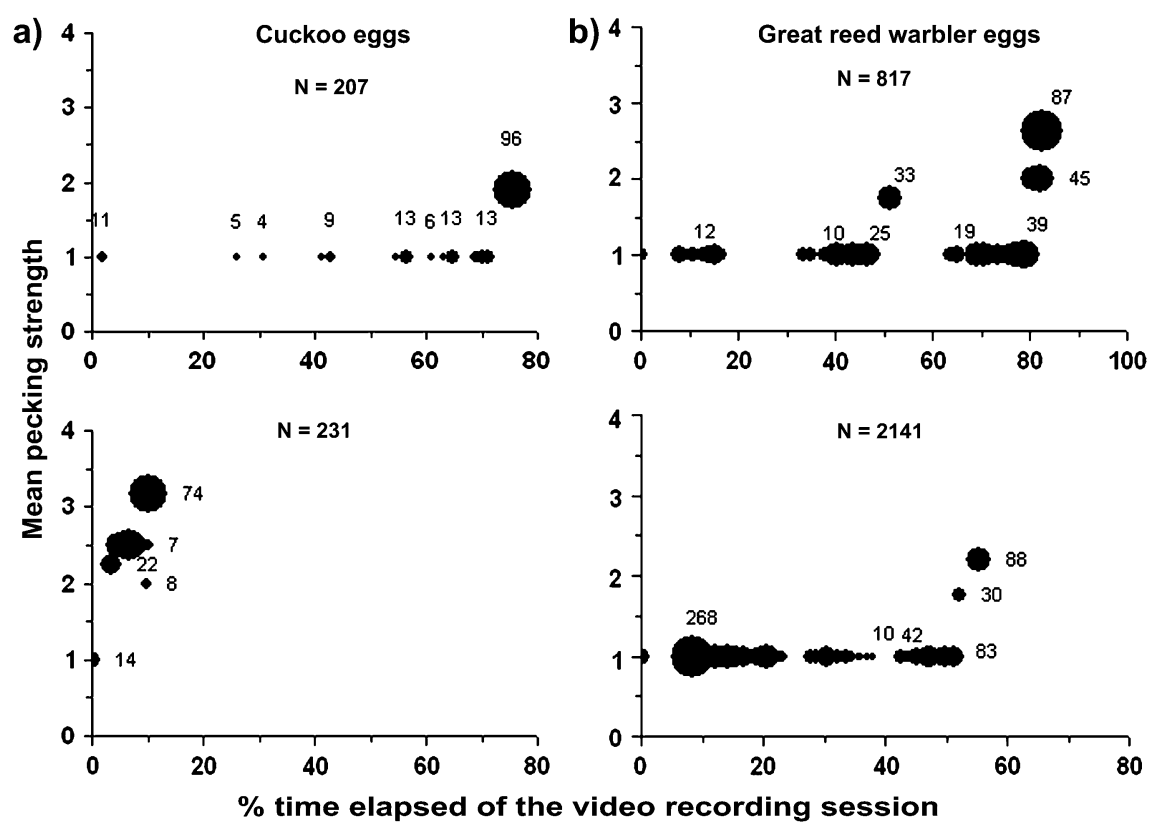

\section{What are the mechanisms behind delayed rejection?}

Our results provided support for prediction (3) regarding the existence of latency to the start of rejection behavior associated with mimetic foreign eggs. Painted eggs were obviously perceived as highly nonmimetic stimuli and triggered rejection behavior more or less immediately after the hosts experienced them. In contrast, unpainted (mimetic) cuckoo eggs did not elicit any pecking response within the first $2 \mathrm{~h}$ of the experiment (one exception only). The existence of latency to the start of rejection behavior in hosts confronted with mimetic eggs was also substantiated by the positive correlation between the day the first pecking was detected and the day the rejection took place. The association of the latency to pecking with mimetic eggs demonstrates that hosts did experience recognition problems and needed time to make a decision if the egg was actually a foreign one.

Furthermore, even after the hosts experiencing mimetic eggs had started pecking, it was less intense than in hosts confronted with nonmimetic eggs (Figure 2). Hosts pecked at a very low rate and only weakly at mimetic eggs on the day the first pecking was recorded. This finding is even more significant when we consider the fact that although the onset of pecking in the 2 nonmimetic treatments was always precisely known (all pecked within the first video recording session), the exact start of pecking in the mimetic treatment was unknown. Thus, when the first pecking was recorded in this treatment, it had most likely continued for some time and perhaps increased in intensity compared with its level within the first $2 \mathrm{~h}$ of its appearance. Nevertheless, after the first pecking in the mimetic treatment was observed, it took these hosts a similar amount of time (in days) to reject the cuckoo egg to those confronted with nonmimetic cuckoo eggs. Therefore, the low pecking effort observed in the mimetic treatment had relatively little effect on the delay to rejection compared with the latency to pecking, perhaps significant only in terms of hours. Because we could not monitor nests continuously until rejection and expressed time to rejection in days, we were unable to detect such fine differences.

These results support the "increasing motivation" hypothesis of Rodrígues-Gironés and Lotem's (1999) for a possible optimal behavioral strategy in hosts confronted with mimetic eggs. The existence of latency to the initiation of rejection behavior suggests that hosts need to inspect their clutches repeatedly and start ejection attempts only after their motivation has exceeded a certain threshold. This involves solving a cognitive problem during which the perception of the foreign egg changes with time and the confidence that it is deviant enough to be considered foreign increases. Such a mechanism seems adaptive as it may reduce the risk of committing recognition errors in host-brood parasite systems in which the parasite has evolved mimetic eggs (see also Stokke et al. 2005). Furthermore, once rejection behavior was released, the level of pecking effort was still kept lower for a certain amount of time than in hosts confronted with nonmimetic eggs. A strategy of a gradual increase in pecking frequency and strength might reduce the probability that the host incidentally pecks some of its own eggs once the aggressive high-rate pecking is underway, that is, rejection errors (sensu Stokke et al. 2002). We did observe how a marsh warbler intensively pecking an unpainted cuckoo egg also destroyed one of its own eggs through a single misdirected strong peck, which was certainly not as a result of the bill ricocheting off the cuckoo egg (Antonov A, Stokke BG, Moksnes A, Røskaft E, unpublished data).

Host pecking effort in the nonmimetic egg treatments, however, indicated that the delay in rejection has an additional component, which was not expected. It is puzzling that even though such hosts obviously did not experience recognition problems, only a small proportion of them rejected the nonmimetic eggs during the first $2 \mathrm{~h}$. Moreover, a considerable proportion of the rejections $(31 \%, 15 / 49)$ took place 2 or more days after the start of the experiment. The strong eggshell of the cuckoo egg explained some of the variation in the time to rejection between the 2 painted egg treatments, but detailed observations on host behavior showed that it was not a major constraint. It was clear on the video recordings, which captured ejections that a pecking marsh warbler needed on average only about 20 and 54 "strong" pecks to puncture a great reed warbler or a cuckoo egg, respectively, in not more than 2 min. Furthermore, the presence of strong pecking within the first $2 \mathrm{~h}$ of the experiment was significantly associated with subsequent rejection during this interval. The pecking profiles of rejecting pairs also showed clearly that puncture was achieved only after hosts sharply increased their 
pecking effort above a certain level (Figure 3). Nevertheless, before effectively increasing their effort, hosts spent a long time producing hundreds and thousands of weak pecks, which were obviously not sufficient to result in a hole in the foreign egg. Our results thus strongly suggest that the factor primarily responsible for the delay in rejection of the nonmimetic eggs was the incidence of weak pecking, not difficulties to puncture the egg. Weak pecking may be an initial exploratory behavior in response to a novel object in the nest. Nonetheless, given that hosts did not experience recognition problems, pecking takes time and energy, and pecking is incompatible with incubation, then prolonged periods of weak pecking resulting in delayed rejection seem suboptimal. One possible explanation for the extensive occurrence of weak pecking is that most host individuals were simply inexperienced at puncture ejecting. Perhaps the majority of the marsh warblers that pecked weakly in most cases were ejecting a foreign egg for the first time, whereas the few birds that showed strong pecking early may have been those that had experienced ejections of a cuckoo egg in a previous breeding attempt. In this sense, ejection abilities may have a learned component. Alternatively, weak pecking might be an adaptive general "warming-up" exercise prior to the effective intensification of pecking leading to puncture ejection if it reduces the risk of own egg damage. However, as discussed above, this seems more relevant in the context of mimetic eggs where a host involved in intensive heavy pecking is at a higher risk of misdirecting some of the pecks at own eggs. Nonmimetic eggs used in this study contrasted sharply to host eggs; thus, the risk of such rejection errors should be minimal, casting doubt on the adaptiveness of weak pecking.

Unfortunately, little can be inferred about the generality and significance of weak pecking because, to our knowledge, no similar studies attempted to quantify pecking strength (Davies and Brooke 1988; Moksnes et al. 1994; Lindholm 2000; MartínVivaldi et al. 2002; Soler et al. 2002). Only Rothstein (1975) and Soler et al. (2002) briefly mentioned soft pecking before ejection of foreign eggs in some hosts but did not discuss this behavior. For better understanding of rejection behavior of hosts of brood parasites, further studies should consider the strength of pecking in addition to other behavioral variables.

\section{FUNDING}

Torstein Erbos Gavefond and the Research Council of Norway (151641/432 to B.G.S.).

We would like to thank Per Harald Olsen for his invaluable help during the fieldwork. Special thanks to Dr Arnon Lotem and an anonymous reviewer for their constructive comments that improved the paper. The present study complied with the current legal regulations of Bulgaria.

\section{REFERENCES}

Alvarez F, Arias de Reyna L, Segura M. 1976. Experimental brood parasitism of the magpie (Pica pica). Anim Behav. 24:907-916.

Amundsen T, Brobakken PT, Moksnes A, Røskaft E. 2002. Rejection of common cuckoo Cuculus canorus eggs in relation to female age in the bluethroat Luscinia svecica. J Avian Biol. 33:366-370.

Antonov A, Stokke BG, Moksnes A, Kleven O, Honza M, Røskaft E. 2006. Eggshell strength of an obligate brood parasite: a test of the puncture resistance hypothesis. Behav Ecol Sociobiol. 60:11-18.

Antonov A, Stokke BG, Moksnes A, Røskaft E. 2006. Egg rejection in marsh warblers (Acrocephalus palustris) heavily parasitized by common cuckoos (Cuculus canorus). Auk. 123:419-430.

Baker ECS. 1942. Cuckoo problems. London: Witherby Ltd.

Brooker MG, Brooker LC. 1991. Eggshell strength in cuckoos and cowbirds. Ibis. 133:406-413.
Cramp S. 1992. Handbook of the birds of Europe, the Middle East and North Africa. Vol. VI: Warblers. Oxford: Oxford University Press.

Davies NB. 2000. Cuckoos, cowbirds and other cheats. London: T \& AD Poyser.

Davies NB, Brooke M de L. 1988. Cuckoos versus reed warblers: adaptations and counteradaptations. Anim Behav. 36:262-284.

Davies NB, Brooke M de L. 1989a. An experimental study of coevolution between the cuckoo, Cuculus canorus, and its hosts. I. Host egg discrimination. J Anim Ecol. 58:207-224.

Davies NB, Brooke M de L. 1989b. An experimental study of coevolution between the cuckoo, Cuculus canorus, and its hosts. II. Host egg markings, chick discrimination and general discussion. J Anim Ecol. 58:225-236.

Davies NB, Brooke M de L, Kacelnik A. 1996. Recognition errors and probability of parasitism determine whether reed warblers should accept or reject mimetic cuckoo eggs. Proc R Soc Lond B. 263: 925-931.

Gärtner K. 1982. Das Wegnehmen von Wirtsvogeleiern durch den Kuckuck (Cuculus canorus). Ornithol Mitt. 33:115-131.

Hays H, LeCroy M. 1971. Field criteria for determining incubation stage in eggs of the common tern. Wilson Bull. 83:425-429.

Honza M, Grim T, Čapek M, Moksnes A, Røskaft E. 2004. Nest defence, enemy recognition and nest inspection behaviour of experimentally parasitized reed warblers Acrocephalus scirpaceus. Bird Study 51:256-263.

Honza M, Picman J, Grim T, Novák V, Čapek M, Mrlík V. 2001. How to hatch from an egg of great structural strength. A study of the common cuckoo. J Avian Biol. 32:249-255.

Honza M, Procházka P, Stokke BG, Moksnes A, Røskaft E, Čapek M, Mrlík V. 2004. Are blackcaps current winners in the evolutionary struggle against the common cuckoo? J Ethol. 22:175-180.

Lindholm AK. 2000. Tests of phenotypic plasticity in reed warbler defences against cuckoo parasitism. Behaviour. 137:43-60.

Lotem A, Nakamura H, Zahavi A. 1992. Rejection of cuckoo eggs in relation to host age-a possible evolutionary equilibrium. Behav Ecol. 3:128-132.

Lotem A, Nakamura H, Zahavi A. 1995. Constraints on egg discrimination and cuckoo host coevolution. Anim Behav. 49:1185-1209.

Lyon B. 2007. Mechanism of egg recognition in defenses against conspecific brood parasitism: American coots (Fulica americana) know their own eggs. Behav Ecol Sociobiol. 61:455-463.

Marchetti K. 2000. Egg rejection in a passerine bird: size does matter. Anim Behav. 59:877-883.

Martín-Vivaldi M, Soler M, Møller AP. 2002. Unrealistically high costs of rejecting artificial model eggs in cuckoo Cuculus canorus hosts. J Avian Biol. 33:295-301.

Mason P, Rothstein SI. 1986. Coevolution and avian brood parasitism: cowbird eggs show evolutionary response to host discrimination. Evolution. 40:1207-1214.

McLean IG, Maloney RF. 1998. Brood parasitism, recognition, and response: the options. In: Rothstein SI, Robinson SK, editors. Parasitic birds and their hosts studies in coevolution. New York: Oxford University Press.

Moksnes A. 1992. Egg recognition in chaffinches and bramblings. Anim Behav. 44:993-995.

Moksnes A, Røskaft E. 1992. Responses of some rare cuckoo hosts to mimetic model cuckoo eggs and to foreign conspecific eggs. Ornis Scand. 23:17-23.

Moksnes A, Røskaft E. 1995. Egg morphs and host preference in the common cuckoo (Cuculus canorus) - an analysis of cuckoo and host eggs from European museum collections. J Zool Lond. 236: $625-648$

Moksnes A, Røskaft E, Bičík V, Honza M, Øien IJ. 1993. Cuckoo Cuculus canorus parasitism on Acrocephalus warblers in Southern Moravia in the Czech Republic. J Ornithol. 134:425-434.

Moksnes A, Røskaft E, Braa AT. 1991. Rejection behavior by common cuckoo hosts towards artificial brood parasite eggs. Auk. 108:348354.

Moksnes A, Røskaft E, Braa AT, Korsnes L, Lampe HM, Pedersen HC. 1990. Behavioral responses of potential hosts towards artificial cuckoo eggs and dummies. Behaviour. 116:64-89.

Moksnes A, Røskaft E, Hagen LG, Honza M, Mørk C, Olsen PH. 2000. Common cuckoo Cuculus canorus and host behaviour at Reed Warbler Acrocephalus scirpaceus nests. Ibis. 142:247-258. 
Moksnes A, Røskaft E, Korsnes L. 1993. Rejection of cuckoo (Cuculus canorus) eggs by meadow pipits (Anthus pratensis). Behav Ecol. 4:120-127.

Moksnes A, Røskaft E, Solli MM. 1994. Documenting puncture ejection of parasitic eggs by chaffinches Fringilla coelebs and blackcaps Sylvia atricapilla. Fauna Norv Ser C Cinclus. 17:115-118.

Moksnes A, Røskaft E, Tysse T. 1995. On the evolution of blue cuckoo eggs in Europe. J Avian Biol. 26:13-19.

Nakagawa S. 2004. A farewell to Bonferroni: the problems of low statistical power and publication bias. Behav Ecol. 15:10441045 .

Øien IJ, Honza M, Moksnes A, Røskaft E. 1996. The risk of parasitism in relation to the distance from reed warbler nests to cuckoo perches. J Anim Ecol. 65:147-153.

Øien IJ, Moksnes A, Røskaft E, Honza M. 1998. Costs of cuckoo Cuculus canorus parasitism to reed warblers Acrocephalus scirpaceus. J Avian Biol. 29:209-215.

Picman J. 1989. Mechanism of increased puncture resistance of eggs of brown-headed cowbirds. Auk. 106:577-583.

Picman J, Pribil S. 1997. Is greater eggshell density an alternative mechanism by which parasitic cuckoos increase the strength of their eggs? J Ornithol. 138:531-541.

Procházka P, Honza M. 2003. Do common whitethroats (Sylvia communis) discriminate against alien eggs? J Ornithol. 144:354-363.

Procházka P, Honza M. 2004. Egg discrimination in the yellowhammer. Condor. 106:405-410.

Rodríguez-Gironés MA, Lotem A. 1999. How to detect a cuckoo egg: a signal-detection theory model for recognition and learning. Am Nat. 153:633-648.
Rohwer S, Spaw CD. 1988. Evolutionary lag versus bill-size constraints: a comparative study of the acceptance of cowbird eggs by old hosts. Evol Ecol. 2:27-36.

Rothstein SI. 1972. Eggshell thickness and its variation in the Cedar Waxwing. Wilson Bull. 84:469-474.

Rothstein SI. 1975. An experimental and teleonomic investigation of avian brood parasitism. Condor. 77:250-271.

Rothstein SI. 1982. Mechanisms of avian egg recognition: which egg parameters elicit host responses by rejecter species? Behav Ecol Sociobiol. 11:229-239.

Rothstein SI, Robinson SK. 1998. Parasitic birds and their hosts: studies in coevolution. New York: Oxford University Press.

Soler M, Martín-Vivaldi M, Pérez-Contreras T. 2002. Identification of the sex responsible for recognition and the method of ejection of parasitic eggs in some potential common cuckoo hosts. Ethology. 108:1093-1101.

Stokke BG, Honza M, Moksnes A, Røskaft E, Rudolfsen G. 2002. Costs associated with recognition and rejection of parasitic eggs in two European passerines. Behaviour. 139:629-644.

Stokke BG, Moksnes A, Røskaft E. 2005. The enigma of imperfect adaptations in hosts of avian brood parasites. Ornithol Sci. 4:17-29.

Stokke BG, Takasu F, Moksnes A, Røskaft E. 2007. The importance of clutch characteristics and learning for anti-parasite adaptations in hosts of avian brood parasites. Evolution. 61:2212-2228.

Underwood TJ, Sealy SG. 2006. Parameters of brown-headed cowbird Molothrus ater egg discrimination in warbling vireos Vireo gilvus. J Avian Biol. 37:457-466.

Zar JH. 1999. Biostatistical analysis. 4th ed. New Jersey (NJ): PrenticeHall, Inc. 